\title{
Anionic surfactant ionic liquids with 1-butyl-3-methyl-imidazolium cations: characterization and application
}

\author{
Paul Brown ${ }^{\dagger}$, Craig Butts ${ }^{\dagger}$, Julian Eastoe ${ }^{\dagger \star}$, David Fermin ${ }^{\dagger}$, Isabelle Grillo ${ }^{\ddagger}$, Huai-Chin Lee ${ }^{\dagger}$, David Parker ${ }^{\dagger}$, \\ Daniela Plana ${ }^{\dagger}$, and Robert Richardson ${ }^{\#}$ \\ ${ }^{\dagger}$ School of Chemistry, University of Bristol, Cantock's Close, Bristol BS8 1TS, U.K., \\ ${ }^{\ddagger}$ Institut Max-von-Laue-Paul-Langevin, BP 156-X, F-38042 Grenoble Cedex, France, \\ ${ }^{\#}$ H. H. Wills Physics Laboratory, University of Bristol, Tyndall Avenue, Bristol BS8 1TS, UK. \\ ${ }^{*}$ corresponding author \\ E-mail: Julian.Eastoe@bristol.ac.uk
}

\section{Abstract}

For the first time a series of anionic surfactant ionic liquids (SAILs) have been synthesized based on organic surfactant anions and 1-butyl-3-methyl-imidazolium cations. These compounds are more environmentally friendly and chemically tuneable as compared to other common ionic liquids. A detailed investigation of physico-chemical properties highlights potential applications from battery design to reaction control, and studies into aqueous aggregation behaviour, as well as structuring in pure ILs, point to possible uses in electrochemistry.

\section{Key Words}

Surfactant ionic liquids (SAILs), mesophase structures, electrochemistry, ionic liquids, small-angle neutron scattering (SANS) 


\section{Introduction}

Ionic liquids (ILs), commonly thought of as liquid salts below $100^{\circ} \mathrm{C}$, are of immense interest as they have physico-chemical properties different from conventional molecular solvents ${ }^{1}$. Typical ILs are 1-butyl-3methyl imidazolium halides (bmim X), and the bmim moiety is a common component in many ILs. Ionic liquids are often considered to be environmentally benign due to low vapour pressure; with up to $10^{9}$ cationanion combinations ${ }^{2}$ they are highly tunable and may be designed for specific chemical and synthetic applications.

It soon became appreciated that long chain analogues of the common cationic imidazolium based ILs could self-assemble to form micelles in aqueous solutions ${ }^{3}$, these systems were then dubbed "surfactant ionic liquids" (SAILs) ${ }^{4}$. Since then catanionic SAILs have been reported ${ }^{5}$, these are novel systems being composed of cations and anions both having an amphiphilic character. Initial studies of cationic SAILs were limited in scope, with only minor chemical variations centred around substituted imidazolium analogues ${ }^{6}$. Here is reported for the first time a systematic study of catanionic SAILs, comprising the 1-butyl-3-methylimidazolium (bmim) cation and common anionic surfactant anions, which also bear bulky hydrophobic chains (Figure 1).

Recently El Seoud et al. ${ }^{7}$ and Eastoe et $a l^{8}$ have shown that the ionic liquid nature confers no special properties in terms of surfactant properties or aggregation behaviour of SAILs: essentially they behave just like regular surfactants. Nonetheless, the development of these SAILs is advantageous as it presents interesting opportunities to combine the properties of surfactants with those of imidazolium-based ILs, and this dual nature may well be beneficial in applications such as separation, condensation and extraction. Recently ${ }^{9}$, it was shown that hydrophobic analytes, such as hydrocarbons, partition strongly into micellized SAILs, suggesting advantages in aqueous phase transfer catalysis, being also appropriate liquid media for extraction and recycling of reagents.

The SAILs reported here are appealing for various reasons. Firstly, aggregation is controlled by the chemical structures of the anions, being more tailorable than the chemically limited sub-set of imidazolium cations commonly employed: in these new systems small structural changes feed through to significant effects on aggregation properties such as critical micelle concentration (cmc), mesophase behaviour, and also on bulk physico-chemical properties such as melting point and solvent miscibility. Secondly, since the compounds contain imidazolium heterocycles it is probable that these SAILs could act as catalysts, either ligating to metal atoms or providing an acidic medium appropriate for certain chemistries (for example 
53 Diels-Alder or Friedel-Crafts reactions) ${ }^{10}$. Thirdly, since they are halogen-free the compounds are more environmentally friendly than traditional imidazolium-based SAILs. In addition to this, the intrinsic nature/properties of the cations and anions selected are also important. For example, anions such as AOT (Figure 1) have antibiotic and pesticidic properties ${ }^{11}$, and imidazolium cations are also known to have antibacterial properties ${ }^{11}$. Another reason for selecting a short alkyl chained imidazolium group is that aquatic toxicity is reduced compared to longer chain analogues ${ }^{12}$.

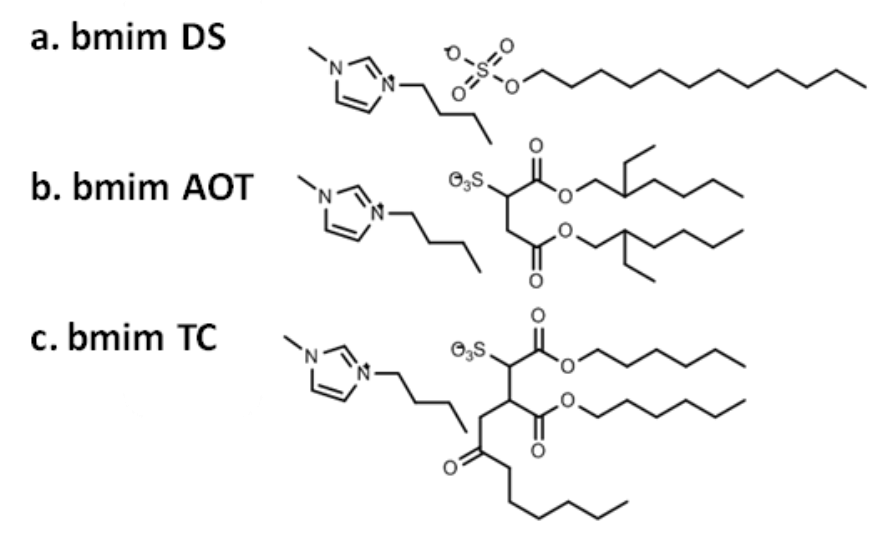

Figure 1: Surfactant ionic liquids (SAILs) studied here

This paper describes the synthesis, chemical characterization (Supporting Information), and physicochemical properties of neat ILs (viscosity, density, melting point, etc.) as well as for aqueous solutions (surface tensiometry, small-angle neutron scattering). Furthermore, a potential application of the SAILs in electrochemistry is explored. Aqueous phase studies are important to learn how self-assembly structure is related to the IL molecular architecture, useful for example in applications where the underlying phase structure can be tuned to provide a new dimension for control over outcomes of organic reactions. Densities are important when considering electrochemical and battery applications, where weight is a concern. In addition, low density may also be advantageous for phase separation of immiscible liquids. Knowledge of viscosity is important for chemical engineering applications, but is also fundamental for uses as chemical reaction media, since $\eta$ affects reactant diffusion coefficients.

Ionic liquids were originally conceived as electrochemical solvents ${ }^{13}$ and are industrially important because they are effective at moderate temperatures, being also less corrosive than classical molten salts. The electrochemical windows of ILs are often $>4 \mathrm{~V}^{14}$, which permits deposition of elements that cannot be delivered from normal aqueous solutions, such as elemental and compound semi-conductors. 
Halogen-free ILs are attractive not only for environmental reasons ( $\mathrm{F}^{-}$can react with water, giving off $\mathrm{HF}$, for example) but also because the presence of $\mathrm{Cl}^{-}$or $\mathrm{Br}^{-}$can affect electrochemical behaviour. In addition, by introducing large organic anions, the hydrophobicity of the ILs may increase, limiting water uptake that can also be detrimental to electrochemical applications. In contrast to variable cathodic potential limits, generally there is little variation in the anodic edge of the potential window, with the most common anions $\left(\mathrm{BF}_{4}^{-}, \mathrm{PF}_{6}^{-}, \mathrm{N}(\mathrm{Tf})_{2}^{-}\right)$lying within a $0.5 \mathrm{~V}$ range ${ }^{13}$. However, this is not the case with the compounds presented here, allowing the possibility to fine tune the anion chemical structure and properties to achieve a much wider electrochemical window than for other available solvent media.

Although, there have been reviews in the field of micellar electrochemistry ${ }^{15}$, the use of SAILs as electrochemical solvents has so far not been investigated. In addition, the combination of surface active IL properties presents intriguing material properties, offering advantages to using microheterogeneous fluids as electrochemical media. Ionic surfactants above the $\mathrm{cmc}$ form full coverage aggregates on metal and carbon electrodes with structures (bilayers, surface micelles) depending on the applied potential, electrode surface and surfactant type ${ }^{16}$, which allows manipulation of surface electrode properties and surface structures. The surfactants solubilise reactants in these structures allowing penetration to the electrode while also excluding water. Hence, the development of SAILs may give rise to both the large potential windows seen for normal ILs and increased surface selectivity fund with surfactants, but now in the same medium. Finally, extremely high capacitances of these new ILs have been determined, which may prove of interest for the future design of supercapacitors and batteries ${ }^{17}$.

This paper described the straightforward synthesis of catanionic SAILs and systematic studies of physicochemical and surface active properties. The results suggest that these SAILs might be replacements for conventional ILs, but also point to new potential applications. 


\section{Experimental}

\section{Materials}

1-butyl-3-methyl-imidazolium chloride ( $\geq 99 \%$ ) was purchased from Sigma-Aldrich and used as received. Sodium dodecylsulfate (SDS) was purchased from Sigma-Aldrich and purified by recrystallization from methanol. Aerosol-OT (AOT) was purchased from Sigma-Aldrich and purified by Soxhlet extraction using dry acetone and subjected to repeated centrifugation ${ }^{18} . \mathrm{D}_{2} \mathrm{O}$ and ethyl acetate $(\geq 99.5 \%)$ were purchased from Sigma-Aldrich and used without further purification.

\section{Synthesis}

Synthesis of the precursor triple-chain Na-TC surfactant (Figure 1) followed the method reported before . $^{8}$ Bmim AOT and bmim TC were synthesized by adding 1.0 mol. eq. of 1-butyl-3-methyl-imidazolium chloride and $\mathrm{Na}-\mathrm{AOT}$ or $\mathrm{Na}-\mathrm{TC}$ in a round-bottom flask and stirring in dichloromethane for 6 hours, the resulting $\mathrm{NaCl}$ precipitate was then filtered off and solvent removed under reduced pressure. The ILs were then dissolved in dry ethyl acetate and centrifuged at $6000 \mathrm{rpm}$ for 1 hour. The pure solution was separated from excess salt, solvent was removed under reduced pressure, and the ILs were then dried in vacuo at $70{ }^{\circ} \mathrm{C}$ for 24 hours. A similar procedure using methanol as solvent was employed to make bmim DS. Bmim AOT and bmim TC were clear liquids, whereas bmim DS was an opaque viscous liquid/solid (depending on cooling rates due to polymorphism). The synthesized surfactant ionic liquids were characterized by elemental analysis and NMR, all being consistent with expected values (Supporting Information, Figure S1, S2, S3 and Table S1).

\section{Karl Fischer Analysis}

Karl Fischer coulometric titration (Metrohm) was used to measure the residual water content of the ILs: analyses were conducted as a function of time over three days, under ambient atmospheric conditions, until no more water was detected.

\section{Differential Scanning Calorimetry (DSC)}

The melting points and thermal behaviour of the ILs were measured using a TA instruments DSC Q200. The heating and cooling ramp for each sample was $10{ }^{\circ} \mathrm{C} \mathrm{min}^{-1}$ and cyclic profiles were repeated three 
times. Example profiles can be found in the Supporting Information, but bmim DS is not included for reasons explained below.

\section{Density Measurements}

Density was measured with an Anton Paar vibrating tube densitometer (DMA 4100), from $25{ }^{\circ} \mathrm{C}$ to $90{ }^{\circ} \mathrm{C}$. The measurements were viscosity corrected and carried out in atmospheric conditions. The instrument was calibrated using ultrapure water (Elga, resistivity = $18 \mathrm{M} \Omega \mathrm{cm}$ ) and atmospheric air, temperatures were controlled to within $\pm 0.01{ }^{\circ} \mathrm{C}$ using a thermostatted water bath.

\section{Viscosity Measurements}

A Bohlin CVO (Malvern Instruments), fitted with a cone and plate $\left(4^{\circ} / 20 \mathrm{~mm}\right)$, was used to determine viscosities between $25-70{ }^{\circ} \mathrm{C}$ (water bath). First, repeat scans of viscosity as a function of shear stress (10$1000 \mathrm{~Pa}$ ) at $25^{\circ} \mathrm{C}$ were carried out to ensure the materials gave linear responses with no shear history. Scans were then repeated at $40{ }^{\circ} \mathrm{C}$ and $70{ }^{\circ} \mathrm{C}$ (Supporting Information). Once Newtonian behaviour was verified, plots of viscosity against temperature were recorded at constant shear stress (50 Pa) (Supporting Information Figure S7 and Table S3). The experiments for bmim DS are not included for reasons given below.

\section{Small-Angle X-Ray Scattering (SAXS)}

Samples were simply placed onto a thin film of mica and measurements recorded with home-built SAXS (Physics Department, University of Bristol), using a sealed-tube $\mathrm{CuK}_{\alpha}$ source with a wavelength of $1.54 \AA$. The scattering pattern covered scattering vectors ranging from $0.01-0.5 \AA^{-1}$.

\section{Polarizing Light Microscopy (PLM)}

A Nikon Optiphot-2 microscope fitted with polarizing filters was used, and images were captured on a PC via a video camera and colour processor connected to the microscope. The liquid crystal phase progression of each SAIL was investigated by the solvent penetration method (i.e. phase cut) ${ }^{8}$. 


\section{Surface Tensions}

Surface tensions $\gamma$ were measured at $25^{\circ} \mathrm{C}$ using the Wilhelmy plate method, on a Krüss $\mathrm{K} 100$ instrument. Glassware was pre-washed with $50 \%$ nitric acid solution and then rinsed thoroughly with distilled water. The Pt plate was cleaned with distilled water and dried in a blue Bunsen flame before each measurement. The cleanliness of the glassware and plate were tested by checking the surface tension of pure water (Elga, resistivity $=18 \mathrm{M} \Omega \mathrm{cm}$ ). For all SAILs, measurements below the $\mathrm{cmc}$ were carried out using low levels of the chelating agent EDTA (99.5\% tetrasodium salt hydrate, Sigma-Aldrich), at constant surfactant to EDTA ratio, in order to sequester trace impurities of divalent cationic species $\left(\mathrm{M}^{2+}\right)$. This is standard practice for obtaining reliable $\gamma$ values with these classes of surfactants (see Supporting Information) ${ }^{18-19}$.

Measurements were repeated at appropriate time intervals to ensure equilibrium. The cmc was taken as the intersection of the two branches of behaviour describing the steep tension decreases, and higher concentration plateaux, respectively. Limiting surface tensions $\left(\gamma_{c m c}\right)$ were also measured by shape analysis of a pendant drop on a DSA10-MK2 instrument (Krüss, Germany), by fitting pendant drop spatial coordinates to the Laplace-Young equation. Interfacial tension measurements between $n$-dodecane and water were made by shape analysis of a pendant drop of water $(\sim 40 \mu \mathrm{L})$ in $4 \mathrm{~mL}$ of $n$-dodecane using the DSA10 MK2.

\section{Small-Angle Neutron Scattering (SANS)}

Scattering was measured on the D22 diffractometer at ILL, Grenoble, France. A neutron wavelength of $\lambda=$ $10 \AA$ was employed at two different detector distances giving $0.0024<Q<0.37 \AA^{-1}$. Data normalization using accepted procedures gave the absolute cross section $\mathrm{I}(Q)\left(\mathrm{cm}^{-1}\right)$ as a function of momentum transfer $Q\left(\AA^{-1}\right)$. Measurements of the dilute aqueous systems ranged from $0.5-4.0 \mathrm{wt} \%$ in $\mathrm{D}_{2} \mathrm{O}$ (scattering length density $\rho=6.33 \times 10^{10} \mathrm{~cm}^{-2}$ ), to provide the necessary contrast, and were placed in Hellma fused silica cuvettes, path-length $2 \mathrm{~mm}$. During data analyses fitting any low level of residual incoherent scattering was accounted for by a small flat background term. Details on data analyses, and scattering laws employed in the model fitting can be found in Supporting Information. Data were analyzed in absolute units $\left(I(Q) / \mathrm{cm}^{-1}\right)$, with the fitted scale factors being consistent with expectations based on the sample compositions. A model for multilayer stacks ${ }^{20}$ was employed (Supporting Information). 


\section{Electrochemical studies}

Measurements of potential windows were carried out at room temperature on pre-dried SAIL samples (vacuum oven). An Autolab PGSTAT-30 potentiostat (Metrohm, Switzerland) was used and the IL medium was contained in a custom built electrochemical cell, designed to accommodate small sample volumes (0.5 $\mathrm{mL}$ ). A silver wire was used as a quasi-reference electrode, with a platinum coil as a counter electrode, and a $25 \mu \mathrm{m}$ diameter gold micro-electrode as the working electrode.

\section{Results and discussion}

\section{Physico-chemical properties}

Karl Fischer analyses revealed that bmim DS contained the highest water levels, with bmim AOT and bmim TC considerably drier (Table 1). The value for bmim AOT is lower than that for pure dry Na-AOT ( 4052 ppm $)^{21}$, possibly due to the larger counterion limiting hydration around the sulfonate group. These values are comparable to that found for a dried standard IL, bmim $\mathrm{BF}_{4}(4538 \mathrm{ppm})^{22}$.

It has been established that residual water content can have a major effect on physico-chemical properties of ionic liquids ${ }^{23}$, however most previous work either fails to mention this or goes to the extreme of carrying out experiments under rather difficult to establish moisture-free conditions ${ }^{24}$. Although the latter is necessary for fundamental research, it does not prove useful for practical applications of ILs. It is in fact impractical to exclude water from the compounds used in this paper owing to water being very difficult to remove in the starting materials (for example, Na-AOT cannot be easily dried to less than $10 \mathrm{~mol} \%$ water $^{21}$ : on the other hand, this structured water may actually be beneficial for certain applications. Recently, Fujita et al. ${ }^{25}$ showed that small amounts of water strongly affect protein solubility in ILs, while retaining the properties of the IL. This is because the water is strongly solvated to the ions, thus limiting its chemical activity.

\begin{tabular}{ccccc}
\hline Compound & $\begin{array}{c}\text { Melting } \\
\text { point } /{ }^{\circ} \mathbf{C}\end{array}$ & $\begin{array}{c}\text { Water content } \\
/ \mathbf{~ p p m}\end{array}$ & $\begin{array}{c}\text { Density at } \mathbf{2 5}{ }^{\circ} \mathbf{C} \\
/\left(\mathbf{g ~ c m}^{-3}\right)\end{array}$ & $\begin{array}{c}\text { Viscosity at 25 } \\
{ }^{\circ} \mathbf{C} \\
/(\mathbf{m P a} \mathbf{~ s})\end{array}$ \\
\hline bmim DS & - & 11790 & - & - \\
bmim AOT & -19 & 3367 & 1.08 & 3916 \\
bmim TC & -22 & 5232 & 1.08 & 3639 \\
\hline
\end{tabular}


The melting points of both bmim AOT and bmim TC are far below room temperature, being $-19{ }^{\circ} \mathrm{C}$ and $-22{ }^{\circ} \mathrm{C}$ respectively, as expected due to the asymmetry and large size of the anions in such ILs. For bmim AOT, a liquid crystal phase is seen up to $25^{\circ} \mathrm{C}$, which melted to yield an isotropic liquid. Other imidazoliumbased ILs with straight chain sulfonate anions have shown this behaviour ${ }^{5}$. It has been suggested that the $\mathrm{C} 2$ proton is essential to promote liquid crystal stabilization ${ }^{6 \mathrm{~b}}$. For the largest anion studied here, the triplechain, TC, no liquid crystal phases were observed within the operating range of the DSC $\left(>-60^{\circ} \mathrm{C}\right)$ (Supporting Information, Figure S4). Profiles for bmim DS were measured with vastly different melting points recorded, probably due to polymorphism and so no firm conclusions could be made about that compound. This is not uncommon behaviour: for example, bmim $\mathrm{Cl}$ has two possible non-interconvertable crystal structures (and melting points) due to the various possible trans gauche arrangements of the cation $^{26}$. Crystallization is promoted when two different local structures (trans and gauche conformers) with different enthalpies of melting transition can coexist. However, for those with similar enthalpies, crystallization is hindered and lower melting are points observed. This may explain the results seen here, and also implies the possibility of polymorphism in the bmim AOT and bmim TC should not be ruled out.

The density $\rho$ values agree with those previously reported for cationic ILs, for which $\rho$ decreases with increasing alkyl chain length substituent on the cation (methylene groups are less dense than imidazole) ${ }^{22}$. (Supporting Information Table S2 and Figure S5). Interestingly, bmim AOT and bmim TC contain high methylene contents so that that their densities $\left(\rho \approx 1.08 \mathrm{~g} \mathrm{~cm}^{-3}\right)$ are much lower than most other ILs. For example, the densities of bmim based ILs have the following densities ${ }^{22}$ (units in $\mathrm{g} \mathrm{cm}^{-3}$ ):

$$
\mathrm{TC}(1.08) \approx \mathrm{AOT}(1.08) \approx \mathrm{Cl}^{-}(1.08)<\mathrm{BF}_{4}^{-}(1.12)<\mathrm{PF}_{6}^{-}(1.36)<\mathrm{Tf}_{2} \mathrm{~N}(1.43)<\mathrm{I}^{-}(1.44)
$$

Small angle x-ray scattering was carried out on the neat SAILs (Supporting Information, Figure S6). Even though the bmim DS sample was isotropic during the experiment, DSC (Supporting Information, Figure S6) showed that the system was polymorphic around this temperature $\left(25^{\circ} \mathrm{C}\right)$. A broad diffraction band is centred around $Q=0.2745 \AA^{-1}(2 \pi / Q=22.88 \AA)$. This peak becomes broader for bmim AOT and bmim TC, suggesting a more disordered structure. The peaks also lower in intensity and shift to higher $Q$ values $(\mathrm{bmim}$ AOT $=21.47 \AA$, bmim TC $=18.83 \AA$ A $)$, consistent with reducing anion lengths $\mathrm{DS}>\mathrm{AOT}>\mathrm{TC}$. Further 
investigation would be required to determine exactly how the anion/cation pair orient relative to each other. However, X-ray data reveal ordering present even in the isotropic liquid phase, which may be important for explaining electrochemical properties such as capacitance (discussed later). Investigation of the structure of "neat" SAILs may also be important for possible applications controlling stereoselective outcomes of organic reactions.

\section{Viscosity measurements}

For bmim DS, shear thinning was observed, possibly due to a structural change (as previously mentioned various polymorphs may be possible). Measurements for bmim DS were not reproducible and so an analogous straight chain surfactant from the literature $\left(\mathrm{bmim} \mathrm{C}_{8} \mathrm{SO}_{4}\right)$ was included in Figure 2 for comparison. The common IL Bmim $\mathrm{PF}_{4}$ was also selected for a more detailed comparison.

Viscosity measurements for the other compounds (bmim AOT and bmim TC) were taken at temperatures ranging from $25-70{ }^{\circ} \mathrm{C}$, with a maximum viscosity of $3640 \mathrm{mPa}$ s reducing to $295 \mathrm{mPa}$ (Figure 2).

Most ionic liquids ${ }^{22}$ may be classed as Newtonian fluids as viscosity remains constant as shear rate increases. This was observed for the most highly branched IL surfactant, bmim TC, even after multiple hystereses of shear stresses had been repeated at increasing temperatures (Supporting Information, Figure S7). Seddon ${ }^{27}$ reported that ILs of the imidazolium $\mathrm{BF}_{4}$ family (cationic surfactants) with longer alkyl chains (typically $n>12$ ) exhibit non-Newtonian behaviour. Surprisingly however, the anionic compounds studied here, with more alkyl carbons than compounds in the previous study ${ }^{27}$, are still Newtonian. For bmim AOT Newtonian behaviour is also seen with the viscosity ranging from $3920 \mathrm{mPa} s$ to $280 \mathrm{mPa} \mathrm{s}$ over the temperature range examined. Astonishingly, the viscosity falls by over $50 \%$ between $25{ }^{\circ} \mathrm{C}$ and $35{ }^{\circ} \mathrm{C}$. These values indicate that branching of the anion causes similar behaviour to that reported in literature, whereby increasing alkyl content on the anion slightly increases viscosity. However, the viscosities are around an order of magnitude higher than similar alkylsulfate based ILs, perhaps owing to an increase in van der Waals interactions owing to long branched alkyl chains, competing with electrostatic terms $^{28}$. An extra factor affecting bulk viscosity may also be hydrogen bonding between the C2 proton and the anionic sulfonate group. An interesting observation is that anion chain branching opens up a much wider window of viscosity, and that this should be tunable depending on the anion chemical architecture. Such control over viscosity may be useful in chemical engineering applications involving reaction kinetics and heat transfer (Figure 2). 


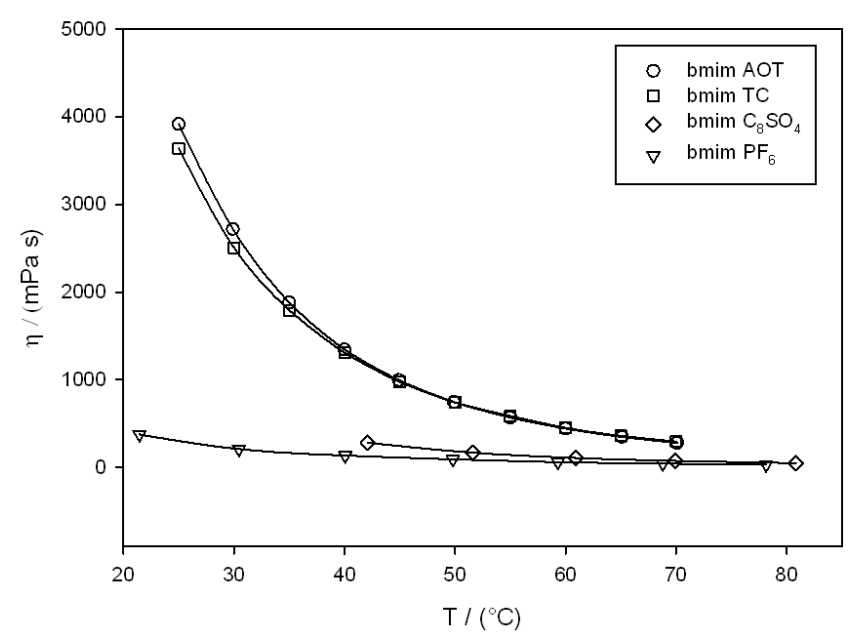

Figure 2: Effect of temperature on viscosity.

Literature data for bmim $\mathrm{PF}_{4}{ }^{29}$ and bmim $\mathrm{C}_{8} \mathrm{SO}_{4}{ }^{30}$ have been included for comparison.

The experimental viscosities were calculated from the integrated form of an Arrhenius-like expression (Equation 1), which is more appropriate than the comparative Vogel-Fulcher-Tammann (VFT) equation, due to the asymmetry and bulk of the anions ${ }^{27}$.

$$
\eta=\eta_{\infty} \exp \left(\frac{E_{\alpha}}{R T}\right)
$$

$\eta_{\infty}$ is the apparent viscosity at infinite temperature and $E_{a}$ is the viscosity activation energy (the semilogarithmic plots are found in the Supporting Information, Figure S8). The magnitude of $E_{a}$ is an indication of the difficulty of transfer of molecules through the liquid matrix. The value for $E_{a}$ with bmim AOT is higher than that for bmim TC (49.46 and $47.08 \mathrm{~kJ} \mathrm{~mol}^{-1}$, respectively), suggesting more order in the bmim AOT system. The activation energies are also higher (approx. 30\%) than those of bmim $\mathrm{PF}_{4}\left(\mathrm{dry}=34.1 \mathrm{~kJ} \mathrm{~mol}^{-1}\right.$, and saturated with $\left.\mathrm{H}_{2} \mathrm{O}=29.0 \mathrm{~kJ} \mathrm{~mol}^{-1}\right)^{29}$ and much higher than for typical molecular solvents (c.f. $\mathrm{H}_{2} \mathrm{O}$ $\left(20^{\circ} \mathrm{C}\right)=17.0 \mathrm{~kJ} \mathrm{~mol}^{-1}$, benzene $=10.4 \mathrm{~kJ} \mathrm{~mol}^{-1}$, and acetone $\left.=7.1 \mathrm{~kJ} \mathrm{~mol}^{-1}\right) .{ }^{31}$ Addition of co-solvents has also been shown to reduce viscosity ${ }^{32}$, and this might be used to provide finer control still. For example, small additions of solvent could affect mesophase formation resulting in larger viscosity changes.

\section{Phase behaviour by polarizing light microscopy (PLM)}

The phase penetration PLM method was used to seek evidence of mesophase formation with added water (Figure 3): different mesophase optical textures appear across the steep concentration gradient set up against water. The transition from fluid micellar to liquid crystalline phases can be explained by a 
competition between increase in free energy associated with loss of orientational entropy, and reduction of free energy related to excluded volume and additional interactions.

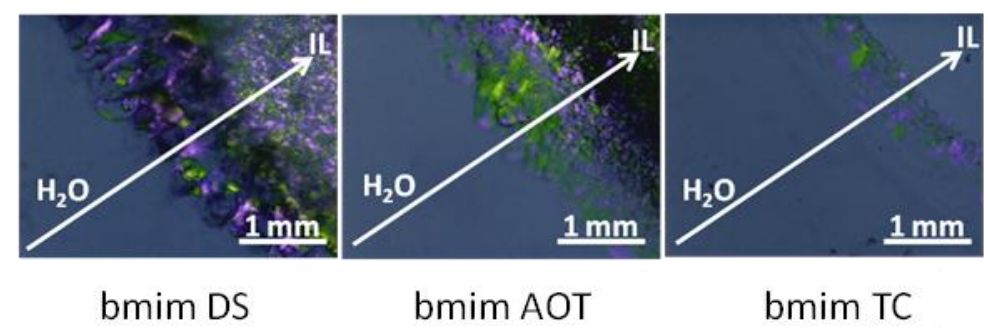

Figure 3: Optical textures of SAILs in phase penetration experiments $\left(\mathrm{T}=25^{\circ} \mathrm{C}\right)$

As mentioned above, the single chained SAIL (bmim DS) exhibits irreproducible properties, possibly because of polymorphism. All batches produced, whether liquid or solid at $25{ }^{\circ} \mathrm{C}$, showed birefringence as neat ILs, evidence of liquid crystalline ordering and mesophase formation in the presence of water. Both bmim AOT and bmim TC showed evidence of mesophase formation on addition of water, but no birefringence in the neat sample, consisitent with findings from DSC and SAXS analyses.

\section{Surface tensiometry and analyses}

Surface tensions as a function of concentration in dilute aqueous solutions were recorded at $25{ }^{\circ} \mathrm{C}$ to establish critical micelle concentrations (cmc) (Figure 4, Table 2, Supporting Information, Table S5), and generate adsorption parameters. Although the self-aggregation behaviour of SAILs has recently attracted much attention, it now appears that in this respect they behave just like regular ionic surfactants ${ }^{7-8}$. For example, the cmcs of the SAILs decrease with increasing carbon numbers on the anion tails, $\mathrm{n}_{\mathrm{c}}$ in broad accordance to the Klevens equation ${ }^{33}$ :

$$
\log (\mathrm{cmc})=A-B n_{c}
$$

where $A$ and $B$ are constants and, for paraffin chain salts having a single ionic headgroup, B is approximately equal to $\log 2$ (i.e., reducing the surface tension to approximately half per each additional $\mathrm{CH}_{2}$ group). It is known that chain branching in hydrocarbon surfactants gives higher cmc values than comparable straight-chain surfactants ${ }^{34}$. 
312 The cmcs for bmim DS and bmim AOT lie lower than their respective normal sodium forms (Table 2$)^{8}$. This 3帒 may be because the bmim cation is more effective at screening intramicellar electrostatic repulsions compared to $\mathrm{Na}^{+6 c}$, but also because bmim is more hydrophobic, thus reducing the free energy of micellization.

The tri-chain SAIL (bmim TC) has a $\mathrm{cmc}$ a little higher than that of its sodium analogue. There are contrasting reports in the literature to how bmim ions might order at an interface ${ }^{35}$. This ordering may be influenced by the structural nature of the anion tails, and in turn affect the ability of the cation to screen headgroup repulsions.
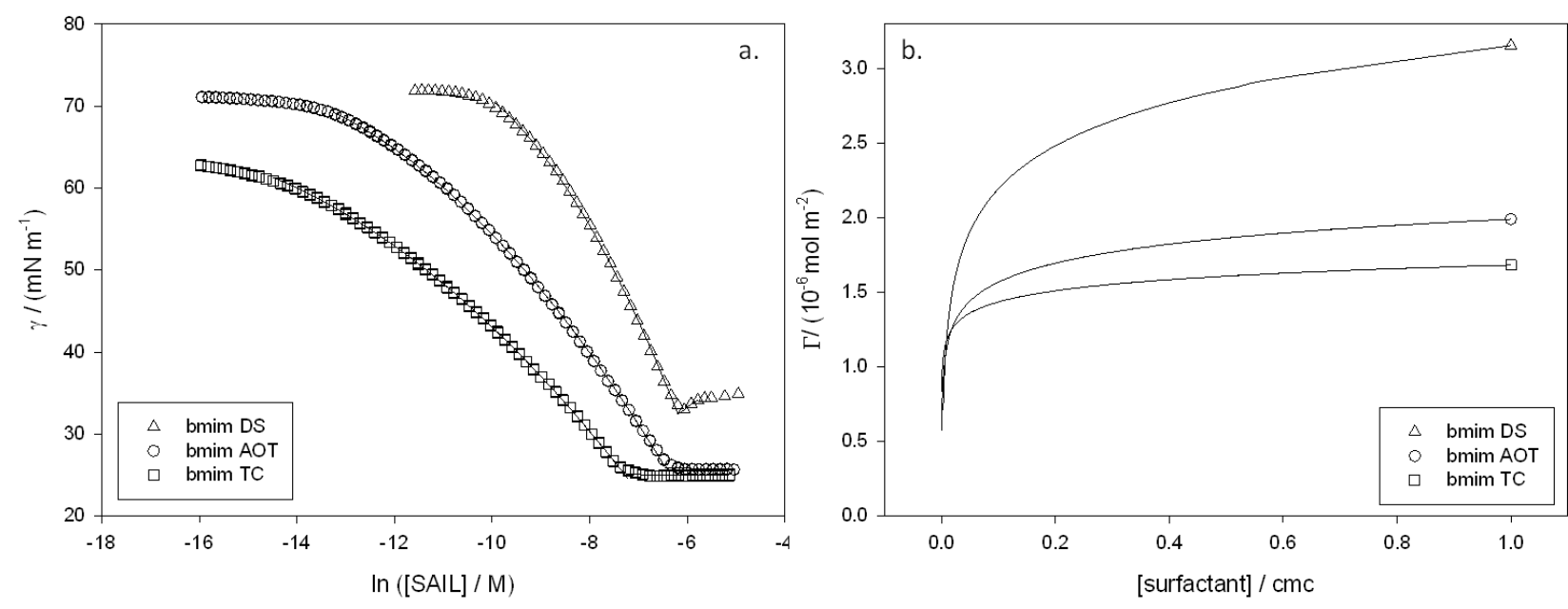

Figure 4: Surface tension data (with quadratic fits to pre-cmc data) and derived adsorption isotherms

\begin{tabular}{lccc}
\hline SAIL & $\begin{array}{c}\mathbf{c m c} / \mathbf{~ m M} \\
\mathbf{\mathbf { 0 . 3 }}\end{array}$ & $\begin{array}{c}\mathbf{Y}_{\mathbf{c m c}} /\left(\mathbf{m N} \mathbf{~ m}^{-1}\right) \\
\mathbf{\mathbf { 0 . 5 }} \\
\text { (Wilhelmy plate) }\end{array}$ & $\begin{array}{c}\mathbf{A}_{\mathrm{cmc}} / \AA^{\mathbf{2}} \\
\mathbf{\mathbf { 1 0 }} \%\end{array}$ \\
\hline Na-DS & 8.00 & 34.9 & 56 \\
Na-AOT & 2.88 & 30.6 & 70 \\
Na-TC & 0.11 & 24.9 & 136 \\
& & & \\
bmim DS & 2.30 & 32.9 & 56 \\
bmim AOT & 1.78 & 25.7 & 86 \\
bmim TC & 0.55 & 24.8 & 111 \\
\hline
\end{tabular}

Table 2: Parameters derived from surface tension measurements.

Data for sodium analogues taken from Eastoe et al. ${ }^{8}$ 
The effectiveness of these ILs at reducing limiting surface tensions is very interesting. It has been observed before that for such SAILs with bulky organic cations, aggregation properties are dominated by anion structure $^{8}$. The single chained bmim DS has a surface tension lower than its sodium analogue $\left(32.9 \mathrm{mN} \mathrm{m}^{-1}\right.$ c.f. $\left.34.9 \mathrm{mN} \mathrm{m}^{-1}\right),{ }^{8}$ as does bmim AOT $\left(25.7 \mathrm{mN} \mathrm{m}^{-1} \text { c.f. } 30.6 \mathrm{mN} \mathrm{m}^{-1} \text { for Na-AOT}\right)^{8}$, with values comparable to those of these anions with tetrapropylammonium cations. However, with bmim TC a lower limiting surface tension is reached, having what is believed to be the lowest surface tension for any imidazolium containing compound. Furthermore, these values are as low as any hydrocarbon surfactant, being comparable to some fluorocarbon surfactants ${ }^{36}$, possibly representing the physical limit of surface tension reduction for such compounds. It is instructive to compare these $\gamma_{c m c}$ values with those for linear alkanes such as hexane $\left(\mathrm{Y}_{\mathrm{cmc}}=18.4 \mathrm{mN} \mathrm{m}^{-1} \text { at } 25^{\circ} \mathrm{C}\right)^{37}$. It has been suggested that the lowest surface tensions are reached when the cation and anion tails are similar in $\operatorname{size}^{5}$, and although this may be true for single chain compounds, for these SAILs there is no further reduction in surface tension (because hydrophobic bulk of the anion governs micellisation) (Supporting information, Figure S10 and Table S5).

The limiting headgroup areas at the $\mathrm{cmc}\left(\mathrm{A}_{\mathrm{cmc}}\right)$ were calculated by fitting pre-cmc tension data to quadratics to generate adsorption isotherms using the Gibbs equation (Equations 4 and 5$)^{18,38}$. A prefactor of $m=2$ is expected for 1:1 dissociating ionic surfactants ${ }^{8}$.

$$
\begin{gathered}
\Gamma=-\frac{1}{\mathrm{mRT} \ln \mathrm{d}} \\
A_{\mathrm{cmc}}=\frac{1}{\Gamma \mathrm{N}_{\mathrm{A}}}
\end{gathered}
$$

Previous studies have shown that for the parent surfactants (Na-DS, Na-AOT, Na-TC)(Table 2), $A_{c m c}$ increases dramatically, consistent with expectations based on packing parameter arguments ${ }^{8}$. The same studies also showed that on replacing the $\mathrm{Na}^{+}$with large bulky tetraalkylammonium cations, $\mathrm{A}_{\mathrm{cmc}}$ was still dominated by the identity of anion, appearing to also be the case with the bmim cation used here. The $A_{c m c}$ value for $\mathrm{Na}-\mathrm{TC}$ is larger than that for the bmim TC but, as mentioned above, this may be a result of ordering of the bmim cation at the air-water interface. 


\section{Micellar structure by SANS}

SANS data were collected as a function of concentration in dilute aqueous phases, with all bmim compounds exhibiting strong $Q^{-2}$ scattering (Figure 5). This scattering is consistent with stacked lamellar phase fragments, and curves could be adequately fit by a model for multilamellar stacks ${ }^{20}$. The fit parameters were $R^{\star}$ (or $\mathrm{R}$ and $\sigma$, where $\mathrm{R}$ is the radius or a multi-walled vesicle and $\sigma$ the layer polydispersity), the local extent of planarity, and $L$, a mean layer thickness of $M$ layers, spaced by $D$ with a superimposed Gaussian distribution of $L$ and $D$ as $\sigma_{L}$ and $\sigma_{D}$, respectively (Figure 6, Table 3).

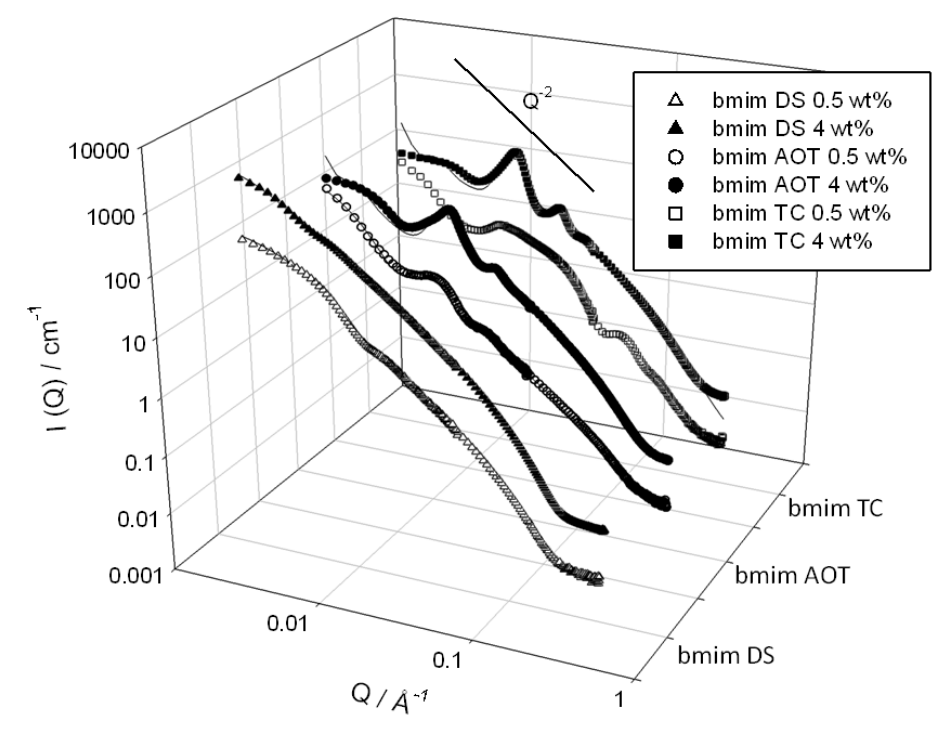

Figure 5: SANS profiles for bmim DS, bmim AOT and bmim TC surfactants in $\mathrm{D}_{2} \mathrm{O}$ at 0.5 and $4 \mathrm{wt} \%$ at $25^{\circ} \mathrm{C}$. Lines through the data are fits using the model for paracrystalline stacks (scattering laws in Supporting Information), with the parameters listed in Table 3. 


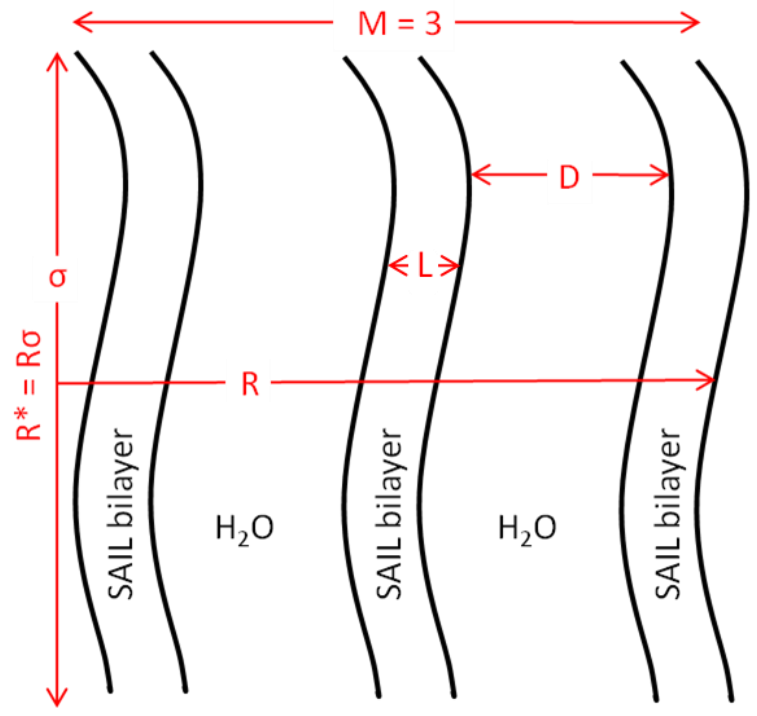

Figure 6: Schematic of paracrystalline stack structure

\begin{tabular}{cccccc}
\hline SAIL & $\mathbf{w t} \%$ & $\mathbf{L} / \mathbf{A}$ & $\mathbf{R}^{*}$ & $\mathbf{M}$ & $\mathbf{D} / \mathbf{A}$ \\
\hline bmim DS & 0.5 & 27.3 & 415 & 2.39 & 136 \\
bmim DS & 1.0 & 30.8 & 223 & 3.47 & 82 \\
bmim DS & 2.0 & 29.2 & 150 & 13.8 & 55 \\
bmim DS & 4.0 & 28.4 & 154 & 15.1 & 68 \\
& & & & & \\
bmim AOT & 0.5 & 12.6 & 676 & 1.9 & 559 \\
bmim AOT & 1.0 & 17.9 & 625 & 1.9 & 559 \\
bmim AOT & 2.0 & - & - & - & $(537)$ \\
bmim AOT & 4.0 & 19.0 & 555 & 6.1 & 462 \\
& & & & & \\
bmim TC & 0.5 & - & - & - & - \\
bmim TC & 1.0 & - & - & - & $(659)$ \\
bmim TC & 2.0 & 21.2 & 529 & 1.8 & 679 \\
bmim TC & 4.0 & 20.5 & 312 & 5.1 & 489 \\
\hline
\end{tabular}

Table 3: Parameters fitted to SANS data using the model for polycrystalline stacks ${ }^{24}$.

Values in brackets estimated using $(2 \pi / Q)$

Bowers et al. ${ }^{3}$ showed that bmim $\mathrm{BF}_{4}$ aggregates above the "cmc" form spherical micelles around $13 \AA$ in radius. It is known that SDS forms spherical micelles ${ }^{8,39}$, and even when substituting sodium for bulky tetraalkylammonium cations the micellar size and shape is maintained ${ }^{8,39}$. Mislkolczy investigated bmim $\mathrm{C}_{8} \mathrm{SO}_{4}$ by turbidity and conductivity measurements, and also observed micelles ${ }^{6 \mathrm{c}}$. Surprisingly, however, at $0.5 \mathrm{wt} \%$ in water this longer $\mathrm{C}_{12}$ chain analogue, bmim DS, forms multilayer stacks where the bilayer thickness $(L)$ is approximately twice the $\mathrm{DS}^{-}$anion length. Increasing the concentration increases the layer 
rigidity $\left(R^{*}\right)$, whilst also increasing the number of bilayers $(M)$. This SANS behaviour for bmim AOT is analogous to other AOT containing $I L s^{8}$, which are known to exhibit a mixed structure $\left(L_{1}+L_{\alpha}\right)$ under these conditions ${ }^{40}$. In comparison to bmim DS, the decrease in bilayer separation is less pronounced, as is the extent of bilayer aggregation, $M$, and the stiffness parameter, $R^{*}$. On increasing the anion size from straight chain to double and triple chain, it appears that the bilayer separation, D, increases substantially. However, none of the other fitted parameters show any obvious trend down the anion series, making it difficult to draw firm conclusions about relationships between anion structure and aggregation.

\section{Electrochemical properties}

Cyclic voltammograms of bmim AOT and bmim TC employing an Au microelectrode at scan rate of $100 \mathrm{mV} \mathrm{s}^{-1}$ are displayed in Figure 7. Taking an arbitrary scale of $100 \mathrm{nA}$, it could be estimated that the potential windows of both SAILs are approximately $4 \mathrm{~V}$. This potential window is significantly wider than those found in aqueous solutions (water $\sim 2 \mathrm{~V}^{41}$ ) and comparable to those reported in conventional 1-butyl3-methyl-imidazolium containing ILs. For example, using a platinum microelectrode, Schröder et al. ${ }^{42}$ reported potential windows of $4.15 \mathrm{~V}$ and $4.10 \mathrm{~V}$ for dry samples of bmim $\mathrm{BF}_{4}$ and bmim $\mathrm{PF}_{6}$ respectively, and Suarez et al..$^{43}$ recorded potential windows of $4.0 \mathrm{~V}$ and $5.0 \mathrm{~V}$. It is also noteworthy that these new ILs have beneficial electrochemical properties, even under anaerobic conditions. Continuous potential cycling of bmim AOT showed progressive passivation of the electrode at high positive potentials (results not shown). This can be observed from the relatively slow rate of current increase at the anodic potential limit (Figure 7). The greater anodic/cathodic current of the bmim TC as compared to bmim AOT could be a result of the lower viscosity (c.f. Figure 2).

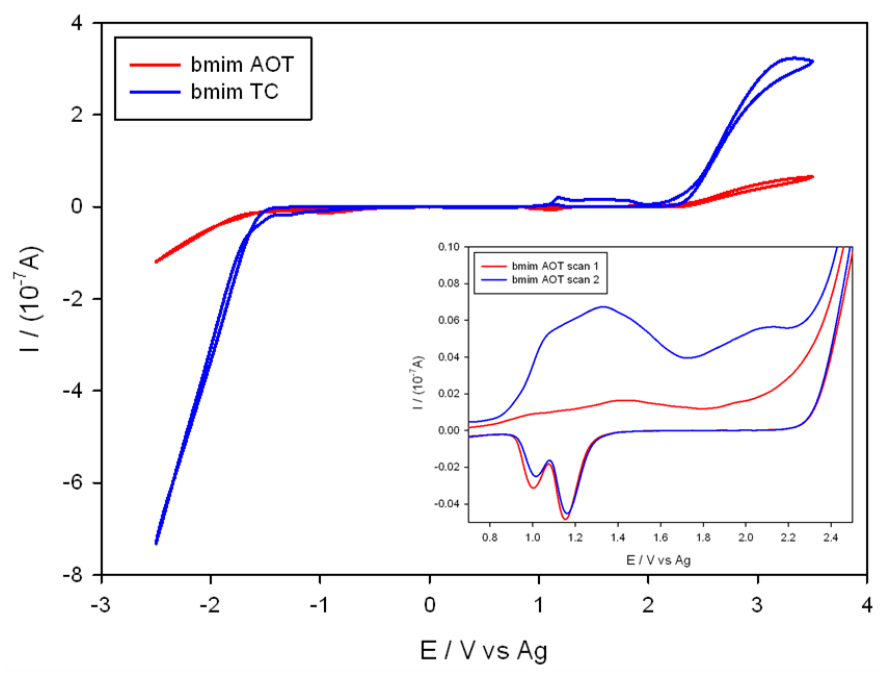

Figure 7: Cyclic voltammograms for bmim AOT and bmim TC on an Au microelectrode 
The anodic responses observed between 1 and $3 \mathrm{~V}$ are linked to the formation of $\mathrm{Au}$ oxide, as well as oxidation of products generated at the negative end of the potential window. As shown in the inset of Figure 7, part of the current responses observed around $1 \mathrm{~V}$ disappear when the potential window is restricted to $-1.5 \mathrm{~V}$. The fact that the reduction products of the imidazolium ring can be oxidised in the reverse scan is likely linked to the high viscosity of the SAILs. Indeed, the hemispherical geometry of the diffusion profiles characteristic of microelectrodes tends to enhance the rate of product diffusion away from the electrode. In this case, the diffusion coefficients are expected to be low, allowing the oxidation of products in the reverse scan. It should also be mentioned that the presence of impurities, such as water, may also be connected to some of the smallest features observed in the voltammograms. A key issue to be investigated in the future is the solubilisation of metal precursors, which could be electrodeposited upon negative polarisation. It is envisaged that structural ordering (Figure 6) could be translated in to the structure of the electrodeposits.

Ionic liquids exhibiting large capacitances offer potential for the development of supercapacitors, which are presently considered to be energy storage devices of choice. The capacitances of these SAILS have been estimated at the ionic liquid-electrode interface (Eq. S3, Supporting Information). Values obtained were 260 $\pm 97 \mu \mathrm{F} \mathrm{cm} \mathrm{cm}^{-2}$ and $34 \pm 12 \mu \mathrm{F} \mathrm{cm}^{-2}$ for bmim AOT and bmim TC respectively. The electric double layer capacitances for bmim $\mathrm{BF}_{4}$ and bmim $\mathrm{PF}_{6}$ have been determined in a detailed study by Lockett et al. ${ }^{44}$ and showed strong potential dependence, with maximum capacitances in the order of $10 \mu \mathrm{F} \mathrm{cm}{ }^{-2}$; significantly less than the capacitances obtained here. Unlike aqueous electrolytes or high-temperature molten salts the ions in ILs are often more complex, with delocalised charges, hydrogen bonding capability and high ion polarizability, leading to microstructuring at the IL-electrode interface. The high capacitances observed here are most probably due to increased amphiphilic nature of the anions, leading to more regular ionic domains (c.f. X-ray scattering data, Supporting Information) and shifting the potential of zero charge (pzc). Conventionally, large anions lead to lower capacitance ${ }^{45}$, but here the anions account for most of the hydrophobic bulk which seems to further increase capacitance. 


\section{Conclusions}

The synthesis and characterization of physico-chemical properties of new SAILs with wide variations in chemical structure have been reported, and potential applications have been explored. The advantages of these compounds over existing ILs and SAILs are facile synthesis: they can also be readily generated from commercially available, cheap starting materials, with lower potential environmental hazards (halogen free) and toxicity (short imidazolium alkyl chain) than normal imidazolium based SAILs. Interestingly, the experimental data obtained under atmospheric conditions suggest suitability of these ILs for electrochemical applications, without the need for excluding air or water, which is a limitation of other common ILs. For example, low densities have been recorded which could be ideal for producing lightweight batteries, and the $50 \%$ drop in viscosity over $10^{\circ} \mathrm{C}$ shows that these ILs may be useful transport media at convenient temperatures.

Polarizing light microscopy shows evidence for mesophase formation, and surface tension measurements show that $\mathrm{cmc}$ can be controlled by hydrophobic bulk of the anion. Furthermore, bmim SAILs can promote very low air-water surface tensions: bmim TC promotes what appears to be the lowest surface tension of any imidazolium based IL, and also of any normal hydrocarbon surfactant, this $\mathrm{Y}_{\mathrm{cmc}}$ of $\sim 25 \mathrm{mN} \mathrm{m}^{-1}$ is equivalent to values of typical low surface energy fluorocarbon analogues. ${ }^{36}$ This could potentially lead to replacement of hazardous fluorinated surfactants, with cheaper more benign hydrocarbon compounds, such as reported here.

In dilute aqueous phases SANS data indicate sheet-like structures for all compounds in dilute aqueous phases, with parameters such as interlayer distances, D, controlled by concentration and anion structure, all consistent with packing parameter arguments ${ }^{46}$. Particularly interesting is how bmim DS follows this trend, deviating from the spherical aggregates seen for other dodecylsulfate anions with large organic counterions, such as the tetraalkylammonium dodecylsulfates ${ }^{8}$.

These results suggest that the ionic liquid nature of bmim SAILs confers no special properties on their aggregation behaviour ${ }^{7-8}$. The unique combination of ionic liquid with surfactant properties may pave the way to the development of novel reaction media, where the possibility of mesophase catalysis may be combined with subsequent separation and extraction from an IL phase. Furthermore, these novel SAILs may find applications in electrochemistry, since the accessible potential window is influenced by the intrinsic ionic liquid nature, with partitioning of reactants at electrodes influenced by the inherent surface 
active properties. Electrochemical results also indicate that these compounds, although containing trace water, still have relatively large potential windows and that this may be further increased through anion substitution. Other possible applications include templating electrodeposition, and due to large capacitances and low densities, in batteries and supercapacitors.

\section{Associated content}

Supporting Information. Additional details of IL synthesis, characterization, surface tension and viscosity measurements, DSC, SANS experiments, and SANS data modelling.

\section{Acknowledgments}

P.B. and D.P. thank the Higher Education Funding Council for England and the School of Chemistry, University of Bristol, for a DTA, Ph.D. scholarship. We also acknowledge the Science \& Technology Facilities Council for the allocation of beam time, travel, and consumables grants at ILL and the Krüss Surface Science Centre for the provision of surface tension facilities. 


\section{$\underline{\text { References }}$}

1. Marsh, K. N.; Boxall, J. A.; Lichtenthaler, R., Fluid Phase Equilibria 2004, 219, 93.

2. Earle, M. J.; Seddon, K. R., Pure Appl. Chem. 2000, 72, 1391.

3. Bowers, J.; Butts, C. P.; Martin, P. J.; Vergara-Gutierrez, M. C.; Heenan, R. K., Langmuir 2004, 20, 2191.

4. El Seoud, O. A.; Pires, P. A. R.; Abdel-Moghny, T.; Bastos, E. L., J. Coll. Int.Sci. 2007, 313, 296.

5. Blesic, M.; Swadzba-Kwasny, M.; Holbrey, J. D.; Canongia Lopes, J. N.; Seddon, K. R.; Rebelo, L. P. N., Phys. Chem. Chem. Phys. 2009, 11, 4260.

6. (a) Wasserscheid, P.; Hal, R. v.; Bosmann, A., Green Chem.y 2002, 4, 400; (b) Mukai, T.; Yoshio, M.; Kato, T.; Ohno, H., Chem. Lett. 2004, 33 (12), 1630; (c) Miskolczy, Z.; Sebok-Nagy, K.; Biczók, L.; Göktürk, S., Chem. Phys. Lett. 2004, 400, 296.

7. Galgano, P. D.; El Seoud, O. A., J.Coll. Int. Sci. 2010, 345, 1.

8. Brown, P.; Butts, C.; Dyer, R.; Eastoe, J.; Grillo, I.; Guittard, F. d. r.; Rogers, S.; Heenan, R., Langmuir 2011, 27, 4563.

9. $\quad$ Yao, C.; Pino, V.; Anderson, J. L., J. Chrom. A 2009, 1216, 948.

10. (a) Yeung, K.-S.; Farkas, M. E.; Qiu, Z.; Yang, Z., Tet. Lett. 2002, 43, 5793; (b) Janus, E.; Goc-Maciejewska, I.; Lozynski, M.; Pernak, J., Tet. Lett. 2006, 47 (24), 4079; (c) Lee, C. K.; Huang, H. W.; Lin, I. J. B., Chem. Commun. 2000, 1911.

11. Hough, W. L.; Smiglak, M.; RodrA?guez, H.; Swatloski, R. P.; Spear, S. K.; Daly, D. T.; Pernak, J.; Grisel, J. E.; Carliss, R. D.; Soutullo, M. D.; Davis, J. J. H.; Rogers, R. D., New J. Chem. 2007, 31, 1429.

12. Cho, C.-W.; Pham, T. P. T.; Jeon, Y.-C.; Vijayaraghavan, K.; Choe, W.-S.; Yun, Y.-S., Chemosphere 2007, 69, 1003.

13. Buzzeo, M. C.; Evans, R. G.; Compton, R. G., Chem. Phys. Chem. 2004, 5, 1106.

14. Rogers, E. I.; Šljukić, B.; Hardacre, C.; Compton, R. G., J. Chem. Eng. Data 2009, 54, 2049.

15. (a) Mackay, R. A.; Texter, J., Electrochemistry in Colloids and Dispersions. VHC: New York, 1992; (b) Bard, A. J.; Stratmann, M., Interfacial Kinetics and Mass Transport. Wiley-VCH: Weinheim, 2003, 2, 553; (c) Rusling, J. F., Acc. Chem. Res. 1991, 24, 75.

16. Rusling, J. F., Coll. Surf. A: Physicochem. Eng. Asp. 1997, 123, 81.

17. Balducci, A.; Bardi, U.; Caporali, S.; Mastragostino, M.; Soavi, F., Electrochem. Commun. 2004, 6, 566.

18. Nave, S.; Eastoe, J.; Penfold, J., Langmuir 2000, 16, 8733.

19. Nave, S.; Eastoe, J.; Heenan, R. K.; Steytler, D.; Grillo, I., Langmuir 2000, 16, 8741.

20. Kotlarchyk, M.; Ritzau, S. M., J. App. Cryst. 1991, 24, 753.

21. Ueda, M.; Schelly, Z. A., Langmuir 1989, 5, 1005.

22. Huddleston, J. G.; Visser, A. E.; Reichert, W. M.; Willauer, H. D.; Broker, G. A.; Rogers, R. D., Green Chem. 2001, 3, 156.

23. Seddon, K. R.; Stark, A.; Torres, M.-J., Pure Appl. Chem. 2000, 72, 2275.

24. Okoturo, O. O.; VanderNoot, T. J., J. Electroanal. Chem. 2004, 568, 167.

25. Fujita, K.; MacFarlane, D. R.; Forsyth, M.; Yoshizawa-Fujita, M.; Murata, K.; Nakamura, N.; Ohno, H., Biomacromolecules 2007, 8, 2080.

26. Hayashi, S.; Ozawa, R.; Hamaguchi, H.-o., Chem. Lett. 2003, 32, 498.

27. Seddon, K. R.; Stark, A. S.; Torres, M.-J., Viscosity and Density of 1-alkyl-3-methylimidazolium ionic liquids. In Ionic Liquids III: Fundamentals, Progress, Challenges, and Opportunities, Rogers, R. D.; Seddon, K. R., Eds. ACS Symposium Series 901, Washington DC: 2004, Vol. 901.

28. (a) Tokuda, H.; Hayamizu, K.; Ishii, K.; Susan, M. A. B. H.; Watanabe, M., J. Phys. Chem. B 2005, 109, 6103; (b) Bonhôte, P.; Dias, A.-P.; Papageorgiou, N.; Kalyanasundaram, K.; Grätzel, M., Inorg. Chem. 1996, 35, 1168.

29. Jacquemin, J.; Husson, P.; Padua, A. A. H.; Majer, V., Green Chem. 2006, 8, 172.

30. Jacquemin, J.; Husson, P.; Majer, V.; Padua, A. A. H.; Costa Gomes, M. F., Green Chem 2008, 10, 944.

31. Wazer, J. R. V.; Lyons, J. W.; Kim, K. Y.; Colwell, R. E., Viscosity and Flow Measurment. Wiley: New York, 1963; 406.

32. (a) Liao, Q.; Hussey, C. L., J. Chem. Eng. Data 1996, 41, 1126; (b) Domańska, U.; Marciniak, A., J. Phys. Chem. B 2004, 108, 2376.

33. Klevens, H., J. Am. Oil Chem. Soc. 1953, 30, 4.

34. Rosen, M. J., Surfactants and Interfacial Phenomena. John Wiley and Sons, Ltd: USA, 1989. 
35. (a) Bhargava, B. L.; Balasubramanian, S., J. Am. Chem. Soc. 2006, 128, 10073; (b) Gannon, T. J.; Law, G.; Watson, P. R.; Carmichael, A. J.; Seddon, K. R., Langmuir 1999, 15, 8429; (c) Rivera-Rubero, S.; Baldelli, S., J. Phys. Chem. B 2006, 110, 4756; (d) Rivera-Rubero, S.; Baldelli, S., J. Phys. Chem. B 2006, 110, 15499; (e) Baldelli, S., J. Phys. Chem. B 2003, 107, 6148; (f) Aliaga, C.; Baldelli, S., J. Phys. Chem. B 2007, 111, 9733.

36. Pitt, A. R.; Morley, S. D.; Burbidge, N. J.; Quickenden, E. L., Coll. Surf. A: Physicochem. Eng. Asp. 1996, 114, 321.

37. Atkins, P.; de Paula, J., Atkins' Physical Chemistry. 7 ed.; Oxford University Press: Oxford, 2002.

38. Eastoe, J.; Downer, A.; Paul, A.; Steytler, D. C.; Rumsey, E.; Penfold, J.; Heenan, R. K., Phys. Chem. Chem. Phys. 2000, 2, 5235.

39. Paul, A.; Griffiths, P. C.; Pettersson, E.; Stilbs, P.; Bales, B. L.; Zana, R.; Heenan, R. K., J. Phys. Chem. B 2005, $109,15775$.

40. (a) Rogers, J.; Winsor, P. A., J. Coll. Int. Sci. 1969, 30, 247; (b) Li, Z. X.; Weller, A.; Thomas, R. K.; Rennie, A. R.; Webster, J. R. P.; Penfold, J.; Heenan, R. K.; Cubitt, R., J. Phys.Chem. B 1999, 103, 10800.

41. Izutsu, K., Electrochemistry in Nonaqeous Solutions. Wiley-VCH: Weinheim, 2002.

42. Schroder, U.; Wadhawan, J. D.; Compton, R. G.; Marken, F.; Suarez, P. A. Z.; Consorti, C. S.; de Souza, R. F.; Dupont, J., New J.Chem. 2000, 24, 1009.

43. Suarez, P. A. Z.; Selbach, V. M.; Dullius, J. E. L.; Einloft, S.; Piatnicki, C. M. S.; Azambuja, D. S.; de Souza, R. F.; Dupont, J., Electrochimica Acta 1997, 42, 2533.

44. Lockett, V.; Horne, M.; Sedev, R.; Rodopoulos, T.; Ralston, J., Phys. Chem. Chem. Phys. 2010, 12, 12499.

45. Costa, R.; Pereira, C. M.; Silva, F., Phys.Chem. Chem.Phys. 2010, 12, 11125.

46. Israelachvili, J. N.; Mitchell, D. J.; Ninham, B. W., J. Chem. Soc., Farad. Trans. 2: Molec. Chem. Phys. 1976, 72, 1525. 
